\title{
Angiotensinogen Gene M235T and T174M Polymorphisms in Patients with Morbid Obesity and Type 2 Diabetes Mellitus
}

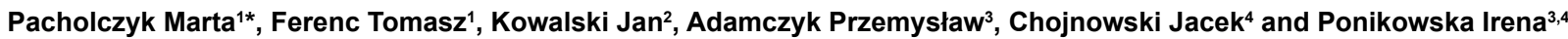

${ }^{1}$ Department of Biology and Medical Genetics, Medical University of Lodz, 1 Haller Sq., 90-647 Lodz, Poland

${ }^{2}$ Department of Internal Diseases and Cardiac Rehabilitation, Medical University of Lodz, 1 Haller Sq. 1, 90-647 Lodz, Poland

${ }^{3}$ Department of Metabolic Diseases, Thermal Hospital in Ciechocinek, Lesna 3 Str., 87-720 Ciechocinek, Poland

${ }^{4}$ Department of Balneology and Physical Medicine, Collegium Medicum in Bydgoszcz, Nicolaus Copernicus University in Torun, Poland

\begin{abstract}
Background: Angiotensinogen and its cleaved form - angiotensin II are important regulators of adipose tissue metabolism and they may affect adipogenesis.

Aim: We studied the correlation between M235T and T174M polymorphisms of angiotensinogen (AGT) gene and morbid obesity and their association with Type 2 Diabetes Mellitus (T2DM). We also investigated the role of haplotypes formed by these polymorphisms in the risk for extreme obesity and obesity- associated T2DM.

Material and methods: The study included 335 morbidly obese patients (study groups) and 230 subjects without obesity (control group). The molecular analysis was performed using Polymerase Chain Reaction (PCR) and PCR-restriction fragment length polymorphism technique.

Results: Distribution of the genotype for AGT M235T polymorphism differed significantly between the controls and all extremely obese patients $(p<0.05)$ and diabetic extremely obese patients $(p<0.05)$. No significant differences were observed for $A G T$ T174M polymorphism between the control and study groups $(p>0.1)$. AGT gene polymorphic variants did not display any difference in clinical or metabolic parameters according to each genotype for either the control or the morbidly obese group. In univariate logistic regression analysis, the carriers for T235T and T174M (Hap2Hap3) had lower risk for extreme obesity $(p<0.05)$. Homozygosity for both T235T and T174T (Hap2Hap2) was associated with decreased risk for extreme obesity and concomitant T2DM $(p<0.05)$.

Conclusion: M235T but not T174M polymorphism of angiotensinogen (AGT) gene, may be linked with extreme obesity and T2DM in the investigated group of patients. On the basis of our results, we suggest that the risk of extreme obesity, irrespective of T2DM, was lower in Hap2Hap3 haplotype pair carriers. The Hap2Hap2 haplotype pair may be a significant protective factor for morbid obesity with T2DM and T2DM development in extreme obesity status $(p<0.05)$.
\end{abstract}

Keywords: $A G T$ gene; Single nucleotide polymorphisms; Haplotype; Genetic susceptibility; Morbid obesity

Abbreviations: T2DM: Type 2 Diabetes Mellitus; RAS: Reninangiotensin System; AGT: Angiotensinogen; SNP: Single-nucleotide Polymorphism; BMI: Body Mass Index; WHR: Waist-to-hip Ratio; SBP: Systolic Blood Pressure; DBP: Diastolic Blood Pressure; OGTT: Oral Glucose Tolerance Test

\section{Introduction}

The prevalence of obesity and $\mathrm{m}$ orbid (extreme) obesity has increased dramatically throughout the recent decades [1]. Due to pathogenic potential of adipose tissue, obesity is associated with many metabolic diseases, including Type 2 Diabetes Mellitus (T2DM), dyslipidemia, hypertension, cardiovascular diseases, fatty liver disease, metabolic syndrome and some forms of cancer [2,3]. Human obesity is a complex quantitative trait that is considered to be influenced by genetic predisposition, environmental and lifestyle factors [4].

Available results of genetic epidemiological studies and genomewide linkage analyses support that alleles in a number of genes contribute to obesity [5]. Little attention has been paid, in literature related to genetic obesity, to genes encoding components of the ReninAngiotensin System (RAS). Increasing evidence suggests that RAS, an important regulator of systemic blood pressure and fluid and electrolyte homeostasis, contributes to the etiology of obesity [6]. In this system, Angiotensinogen (AGT), the unique precursor of angiotensin II (Ang
II), and other bioactive angiotensin peptides, undergo two enzymatic cleavages by renin and Angiotensin Converting enzyme (ACE) to produce angiotensin I (Ang I) and angiotensin II (Ang II), respectively. It has been reported that $A G T$ gene and other components of the RAS are expressed in and have independent regulation of adipose tissue and that adipose tissue has the ability to synthetize Ang II independent of systemic RAS. Mature adipocytes express all components of the RAS, including angiotensinogen (AGT), and chymase. Adipocytes also express type 1 (AT1) and type 2 (AT2) angiotensin receptor subtypes $[7,8]$. The local adipose RAS exerts important auto/paracrine functions in modulating lipogenesis, lipolysis, adipogenesis as well systemic and adipose tissue inflammation. There is also emerging evidence for a role

*Corresponding author: Pacholczyk Marta, Department of Biology and Medical Genetics, Medical University of Lodz, 1 Haller Sq., 90-647 Lodz, Poland, Tel: 48 4263933 43; Fax: 484263933 41; E-mail: marta.pacholczyk@umed.lodz.pl, marta-mp17@o2.pl

Received August 12, 2014; Accepted December 14, 2014; Published December 16, 2014

Citation: Marta P, Tomasz F, Jan K, Przemysław A, Jacek C, et al. (2015) Angiotensinogen Gene M235T and T174M Polymorphisms in Patients with Morbid Obesity and Type 2 Diabetes Mellitus. J Diabetes Metab 6: 479. doi:10.4172/21556156.1000479

Copyright: (C) 2015 Marta P, et al. This is an open-access article distributed under the terms of the Creative Commons Attribution License, which permits unrestricted use, distribution, and reproduction in any medium, provided the original author and source are credited. 
of RAS in regulation of resting metabolic rate, glucose homeostasis and other key metabolic parameters. Since adipose RAS is over activated in many obesity conditions, it is considered a potential candidate linkong obesity to insuline resistance, hypertension, and other metabolic derangements [6,9-11].

Several studies in humans and animals demonstrated that obesity was associated with enhanced activity of both systemic and adipose tissue RAS. Particularly the AGT synthesis was much developed in adipocytes and contributed significantly to the systemic pool. It was shown that plasma AGT concentrations were positively correlated with Body Mass Index (BMI) in human subjects and that the activity of tissue RAS was higher in visceral adipose tissue than in subcutaneous tissue $[9,10,12]$. AGT and its cleaved form - angiotensin II participated in the process of differentiation of pre-adipocytes into adipocytes and in the development of cell hyperplasia (increase in the number of fat cells) and/or hypertrophy (expansion of lipid volume within a fat cell) observed in extreme obesity $[9,13]$. Adipocyte hypertrophy has been associated with insulin resistance, whereas adipocyte hyperplasia has been associated with improved glucose tolerance due to increased metabolic activity in the newly produced adipocytes. Ang II has been shown to influence both these parameters [14]

Angiotensinogen and its cleaved form - Ang II are important regulators of adipose tissue RAS. The gene for angiotensinogen, $A G T$, is characterized by two common single-nucleotide polymorphisms (SNPs) M235T - substitution of methionine (M) to threonine (T) at position 235 (NCBI SNP ID: rs4762) and T174M - substitution of threonine $(\mathrm{T})$ to methionine $(\mathrm{M})$ at position 174 of the mature protein (NCBI SNP ID: rs699) [15]. Both AGT polymorphisms are located in exon 2 [15] and have been found to be associated with angiotensinogen levels [16]. They have been the subject of intensive investigations with regard to hypertension, cardiovascular diseases, type 2 diabetes and obesity but the results are inconsistent [17-22]. Some reports showed an interaction between $A G T$ gene and BMI or/and T2DM [23-28], whereas others did not $[21,29,30]$. In world literature, no reports have been published that examined the correlation between AGT M235T and T174M polymorphisms and morbid obesity. Since no studies have explored the potential interaction between these genetic variants and risk for morbid obesity and concomitant type 2 diabetes mellitus, our study may be considered pioneer in this field of knowledge. We previously reported that $A C E$ gene insertion/deletion (I/D) polymorphism, but not AGTR1 gene $A 1166 C$ polymorphism, could be associated with the risk of extreme obesity and T2DM [31]. To extend those findings [31], we investigated the associations of M235T and T174M polymorphisms of AGT gene with morbid obesity. We also wanted to explore the impact of the two $A G T$ substitution polymorphisms on T2DM development in the group of extremely obese patients. Furthermore, it was also the aim of the present study to broaden these investigations by analyzing the haplotypes as potential risk factors for morbid obesity and T2DM in Polish patients.

\section{Materials and Methods}

\section{Study subjects}

A group of 335 patients (217 females, 64.78\%; 118 males, 35.22\%), with extreme obesity (BMI $\geq 40 \mathrm{~kg} / \mathrm{m}^{2}$ ) were enrolled into the study. The mean age in that group was $54.13 \pm 11.98$ years (mean \pm standard deviation - SD). Diabetes mellitus was diagnosed according to the criteria of the American Diabetes Association [32]. Type 2 diabetes mellitus (non-insulindependent diabetes) is a metabolic disease, which encompasses individuals who have insulin resistance and usually have relative insulin deficiency. Type 2 diabetes is diagnosed on the basis on clinical symptoms of hyperglycemia and biochemical parameters. Criteria for the diagnosis of diabetes mellitus according to American Diabetes Association were: $\mathrm{A} 1 \mathrm{C} \geq 6.5 \%$ or fasting plasma glucose (FPG) $\geq 126 \mathrm{mg} / \mathrm{dL}(7.0 \mathrm{mmol} / \mathrm{L})$ or two hour plasma glucose $\geq 200$ $\mathrm{mg} / \mathrm{dL}(11.0 \mathrm{mmol} / \mathrm{L})$ during oral glucose tolerance test (OGTT) [32].

In our study, individuals with a history of type 2 diabetes or treatment with oral antidiabetics or insulin were considered as subjects with diabetes. Basing on the diagnosed T2DM in the group of patients with extreme obesity, two subgroups were distinguished: without T2DM $(n=134)$ and with T2DM $(n=201)$. The control group comprised 230 normal weight $\left(\mathrm{BMI} \leq 25 \mathrm{~kg} / \mathrm{m}^{2}\right)$ or overweight (BMI $25-29.5 \mathrm{~kg} /$ $\mathrm{m}^{2}$ ) subjects (mean age $49.90 \pm 16.30$ years; 152 females, $66.09 \%$ ), recruited from healthy population undergoing a routine health checkup, who had never been obese. The study included only those patients for whom complete clinical and biochemical data were collected and genomic DNA was obtained for genetic determination.

All the patients and controls were native, unrelated inhabitants of Poland and they were recruited from the Clinic of Balneology and Metabolic Disorders of Thermal Hospital in Ciechocinek, Poland (from November 2010 to March 2014). Exclusion criteria, as in our earlier study [31], were the following: secondary form of obesity, type 1 diabetes mellitus, renal, hematologic, hepatic and thyroid diseases, evidence of other metabolic diseases and corticosteroid therapy. Most of the patients took antihypertensive and lipid lowering drugs, which was not considered as exclusion criteria.

The study protocol was approved by the Bioethical Committee of the Medical University of Lodz (No. RNN/656/10/KB; 16 November, 2010). An informed written consent was obtained from each participant after full explanation of the scientific purposes of the research project and the nature of all used procedures.

\section{Measurements and biochemical analyses}

All subjects underwent anthropometric measurements (weight and height) in the fasting state and lightweight clothes using standard anthropometric techniques. Body Mass Index $\left(\mathrm{kg} / \mathrm{m}^{2}\right)$ was calculated. Waist circumference was measured with a tape measure at a level midway between the lower rib margin and the iliac crest (the greatest constriction of the trunk) in women and at navel level in men. The measurements were performed during apnea. Hip circumference was measured at the widest part of the hips in women and at the upper end of the ilium in men. Waist-to-hip ratio (WHR) was defined as the ratio between the circumferences of the waist to the hip. Extreme obesity was defined as BMI $\geq 40 \mathrm{~kg} / \mathrm{m}^{2}$, according to the recommendations of the World Health Organization [33]. Blood pressure (BP) readings were taken in sitting position after resting for at least 15 min using a standard sphyngomanometer on the left arm. Systolic Blood Pressure (SBP) and Diastolic Blood Pressure (DBP) were measured according to Korotkoff phase I and phase V, respectively. The mean $\mathrm{BP}$ value was calculated from 2-6 measurements. All biochemical and anthropometric measurements were taken during a 3-week stay at the inpatient ward of the Clinic of Balneology and Metabolic Disorders of Thermal Hospital in Ciechocinek.

Basic clinical characteristics, including Total Cholesterol (TC), Triglycerides (TG), High Density Lipoprotein-Cholesterol (HDL-C), Low-Density Lipoprotein-Cholesterol (LDL-C) and glucose were measured in all subjects in the fasting state. The serum concentrations of TC, TG, HDL-C HDL-C LDL-C were measured using Randox reagents and an auto-analyzer. Total cholesterol and triglyceride assays 
were based on colorimetric method. A direct enzymatic technique was used for HDL and LDL cholesterol estimation. Oral Glucose Tolerance Test (OGTT) was also performed in all extremely obese patients according to clinical recommendations ( 2 hours after $75 \mathrm{~g}$ oral glucose load). Information regarding age, gender, smoking habit, duration of diabetes, type of treatment and family history of obesity and diabetes was obtained during a baseline examination. Furthermore, a general health questionaire related to personal and family history of the disease and lifestyle factors was completed.

\section{Genotyping}

Peripheral blood samples were collected from all of the patients and controls into ethylene diamine tetraacetic acid (EDTA)-containing tubes. Genomic DNA was extracted from $200 \mu$ of peripheral blood leukocytes with the use of DNA isolation kit (GeneJET ${ }^{\mathrm{Tax}}$ Genomic DNA Purification Kit, Fermentas, Vilnius, Lithuania), according to the manufacturer's protocol.

The AGT M235T (704T/C) and T174M (521C/T) polymorphisms [17] were investigated by Polymerase Chain Reaction (PCR) amplification of genomic DNA followed by restriction endonuclease digestion. The primers for PCR amplification of AGT M265M polymorphism were: forward [sense] F 5' - CCG TTT GTG CAG GGC CTG GCT CTC T - 3' and reverse [antisense] R 5' - GCC AGG GTG CTG TCA CAC TGA CTC CC - 3' [34]. PCR was performed with thermal cycler and thermostable Taq polymerase (Fermentas, Vilnius, Lithuania), in a final volume of $25 \mu \mathrm{l}$ reaction mixture containing 100 ng of genomic DNA as template, $50 \mathrm{pM}$ of each primer, $0.5 \mu \mathrm{M}$ of each dNTP, $1 \mathrm{U}$ of Taq polymerase, buffer PCR 10x and distilled water. DNA fragments were amplified for 35 cycles, cycling conditions were: denaturation at $94^{\circ} \mathrm{C}$ for $60 \mathrm{~s}$, annealing $64 \mathrm{oC}$ for $40 \mathrm{~s}$ and extension at $72^{\circ} \mathrm{C}$ for $40 \mathrm{~s}$ after initial denaturation at $94^{\circ} \mathrm{C}$ for $5 \mathrm{~min}$ and with final 10-minute elongation. The $165 \mathrm{bp}$ PCR products were digested with the $1 \mathrm{U}$ restriction enzyme BoxI (Fermentas, Vilnius, Lithuania) at $37^{\circ} \mathrm{C}$ for 10 to $30 \mathrm{~min}$ (Fast Digest) and electrophoresed on a $3 \%$ agarose gel stained with ethidium bromide, and visualized under UV light. The $M$ allele was displayed as a single uncleaved band of $165 \mathrm{bp}$. In the presence of $T$ allele, the cleavege yielded two fragments of 141 and $24 \mathrm{bp}$, and only $141 \mathrm{bp}$ band was visualized.

The primers for PCR amplification AGT T174M polymorphism were: forward [sense] F 5' - TAC AGG CAA TCC TGG GTG TTC CTT G - 3' and reverse [antisense] R 5' - AGC AGA GAG GTT TGC CTT ACC TTG - 3' [35]. PCR was performed under the same conditions except for an annealing temperature of $58^{\circ} \mathrm{C}$. The $405 \mathrm{bp}$ PCR products were digested with endonuclease $\mathrm{NcoI}$ (Fermentas, Vilnius, Lithuania) with $1 \mathrm{U}$ enzyme at $37^{\circ} \mathrm{C}$ for 10 to $30 \mathrm{~min}$ (Fast Digest). The digested fragments were electrophoresed in $3 \%$ agarose gels that were stained with ethidium bromide. The undigested fragment (405 bp) represented the T174 allele, while two cleavaged fragments (259 bp and $146 \mathrm{bp}$ ) represented M174 allele.

To certify genotyping quality, all polymorphisms were regenotyped in $10 \%$ randomly selected samples. The check confirmed the previous genotyping results by $100 \%$.

\section{Statistical analysis}

Quantitative variables (anthropometric and biochemical measures) were expressed as mean and Standard Deviation (SD) and differences between groups were assesed with Student's $t$ test. The minimum study sample size was calculated using the power analysis for $\chi^{2}$ tests for multiple proportions, estimating the expected effects from the pilot data and assuming the alpha level of 0.05 and the power of $90 \%$. HardyWeinberg equilibrium was evaluated on the basis of the expected genotype distribution by the $\chi^{2}$ test.

Statistical differences of genotype distributions and allele frequencies of the $A G T$ genotypes between patients with extreme obesity and the control group were assesed by the $\chi 2$ test or Fisher's exact test when the total number of observations was $<5$. To find out the effect of genotypes and haplotypes on the extremely obese and on T2DM status, the logistic regression analysis was performed at the univariate level and the odds ratio (OR) was calculated with $95 \%$ confidence interval (CI).

The correlations of the $A G T$ gene polymorphisms with continuous variables were tested using one-way ANOVA. In all the analyses, $p<$ of 0.05 was considered as the level of statistical significance. STATISTICA 10.0 PL was used for all calculations.

\section{Results}

The clinical and biochemical characteristics of the patients with extreme obesity and of the lean control subjects are presented in Table 1. The morbidly obese group consisted of 335 patients. As expected, significant differences between study and control subjects were found in most diagnosed parameters, whereas there was no statistically significant difference in gender proportion between the investigated groups (Table 1).

The genotype frequencies of $A G T$ M235T polymorphism were in accordance with the Hardy-Weinberg equilibrium (HWE) $(p>0.1)$ in controls and in subjects without T2DM $(p>0.1)$. Deviation from HWE was found in all extremely obese subjects $(\chi 2=7.829, p=0.02)$ and also in patients with extreme obesity and T2DM $(\chi 2=8.77, p=0.012)$. The distribution of $A G T$ T174M genotypes was compatibile with HardyWeinberg expectations in all investigated groups $(p>0.1)$. The genotype and allele frequencies of both examined AGT gene polymorphisms in extremely obese patients and healthy subjects are depicted in Table 2. All clinical and biochemical parameters did not differ between the AGT M235T and T174M genotypes of the total study population (data not shown). Similar observations were made in subgroups of extremely obese patients without or with T2DM ( $p>0.1)$ (data not shown).

\begin{tabular}{|c|c|c|c|}
\hline & $\begin{array}{c}\text { Control subjects } \\
(\mathbf{n}=\mathbf{2 3 0})\end{array}$ & $\begin{array}{c}\text { Extremely obese } \\
\text { patients } \\
\mathbf{( n = 3 3 5 )}\end{array}$ & $\boldsymbol{p}$ value \\
\hline Age [years] & $49.90 \pm 16.30$ & $54.13 \pm 11.98$ & $<0.001$ \\
\hline Male/female [\%] & $33.91 / 66.09$ & $35.22 / 64.78$ & 0.955 \\
\hline BMI [kg/m $]$ & $24.04 \pm 2.33$ & $44.82 \pm 5.51$ & $<0.001$ \\
\hline WHR: Women & $0.7971 \pm 0.0787$ & $0.9475 \pm 0.0720$ & $<0.001$ \\
\hline WHR: Men & $0.9063 \pm 0.0875$ & $1.0217 \pm 0.0613$ & $<0.001$ \\
\hline Hypertension [\%] & 33.33 & 88.89 & $<0.001$ \\
\hline SBP [mm Hg] & $115.60 \pm 26.53$ & $123.67 \pm 8.86$ & $<0.001$ \\
\hline DBP [mm Hg] & $70.72 \pm 21.32$ & $78.52 \pm 3.95$ & $<0.001$ \\
\hline Fasting glucose [mg/dL] & $95.37 \pm 12.84$ & $120.90 \pm 33.05$ & $<0.001$ \\
\hline T2DM \% & 0 & 60.00 & $<0.001$ \\
\hline TC $[\mathrm{mg} / \mathrm{dL}]$ & $207.45 \pm 46.38$ & $203.39 \pm 57.23$ & $<0.05$ \\
\hline LDL-C [mg/dL] & $124.39 \pm 40.52$ & $117.00 \pm 43.12$ & $<0.05$ \\
\hline HDL-C [mg/dL] & $63.14 \pm 17.58$ & $52.93 \pm 16.65$ & $<0.001$ \\
\hline TG [mg/dL] & $110.72 \pm 66.54$ & $153.02 \pm 75.27$ & $<0.001$ \\
\hline
\end{tabular}

Data are presented as mean \pm SD (standard deviation), BMI: Body Mass Index WHR: Waist To Hip Ratio; SBP: Systolic Blood Pressure; DBP: Diastolic Blood Pressure; T2DM: Type 2 Diabetes Mellitus; TC: Total Cholesterol; LDL-C: LowDensity Lipoproteins; HDL-C: High-Density Lipoproteins; TG: Triglicerydes.

Table 1: Clinical and biochemical characteristics of the extremely obese patients and control subjects. 
Citation: Marta P, Tomasz F, Jan K, Przemysław A, Jacek C, et al. (2015) Angiotensinogen Gene M235T and T174M Polymorphisms in Patients with Morbid Obesity and Type 2 Diabetes Mellitus. J Diabetes Metab 6: 479. doi:10.4172/2155-6156.1000479

Page 4 of 8

\begin{tabular}{|c|c|c|c|c|c|}
\hline & \multicolumn{2}{|c|}{$\begin{array}{l}\text { All Patients with genotype or } \\
\text { allele }(\%[\mathrm{n}])\end{array}$} & \multicolumn{2}{|c|}{$\begin{array}{l}\text { Extremely obese } \\
\text { patients with genotype } \\
\text { or allele }(\%[\mathrm{n}])\end{array}$} & \\
\hline & $\begin{array}{l}\text { Controls }(n= \\
230)\end{array}$ & $\begin{array}{l}\text { Extremely obese } \\
\text { patients }(n=335)\end{array}$ & $\begin{array}{c}\text { without } \\
\text { T2DM }(n= \\
134)\end{array}$ & $\begin{array}{l}\text { with T2DM } \\
(\mathrm{n}=201)\end{array}$ & \\
\hline \multicolumn{6}{|l|}{ M235T } \\
\hline$M M$ & $28.70[66]$ & 29.55 [99] & 32.84 [44] & $27.36[55]$ & \\
\hline$M T$ & $52.17[120]$ & 58.81 [197] & 52.99 [71] & 62.69 [126] & \\
\hline \multirow[t]{2}{*}{$T T$} & 19.13 [44] & 11.64 [39] & 14.18 [19] & $9.95[20]$ & \\
\hline & $p$ value & $0.04265^{a}$ & $0.42951^{b}$ & $0.01631^{\circ}$ & $0.18865^{d}$ \\
\hline Allel $M$ & 54.78 [252] & 58.96 [395] & 59.33 [159] & $58.71[236]$ & \\
\hline \multirow[t]{2}{*}{ Allel $T$} & $45.22[208]$ & 41.04 [275] & 40.67 [109] & $41.29[166]$ & \\
\hline & $p$ value & $0.164^{a}$ & $0.233^{b}$ & $0.246^{c}$ & $0.873^{d}$ \\
\hline \multicolumn{6}{|l|}{ T174M } \\
\hline$T T$ & $73.91[170]$ & $70.45[236]$ & 72.39 [97] & 69.15 [139] & \\
\hline$T M$ & 25.22 [58] & 28.06 [94] & 26.87 [36] & $28.86[58]$ & \\
\hline \multirow[t]{2}{*}{ MM } & 0.87 [2] & $1.49[5]$ & $0.75[1]$ & $1.99[4]$ & \\
\hline & $p$ value & $0.602^{\mathrm{a}}$ & $0.912^{b}$ & $0.418^{c}$ & 0.58680 \\
\hline Allel $T$ & 86.52 [398] & 84.48 [566] & $85.82[230]$ & 83.58 [336] & \\
\hline \multirow[t]{2}{*}{ Allel $M$} & $13.48[62]$ & $15.52[104]$ & $14.18[38]$ & $16.42[66]$ & \\
\hline & $p$ value & $0.340^{\mathrm{a}}$ & $0.791^{\mathrm{b}}$ & $0.226^{c}$ & $0.433^{d}$ \\
\hline
\end{tabular}

$p$ values indicate differences in genotype and allele frequencies between groups: ${ }^{a}$ controls vs. all cases; ${ }^{b}$ controls vs. cases without T2DM; ${ }^{\circ}$ controls vs. cases with T2DM; ${ }^{d}$ cases without T2DM vs. cases with T2DM

AGT: Angiotensinogen; T2DM: Type 2 Diabetes Mellitus

Table 2: Genotypes of the angiotensinogen (AGT) gene M235T and T174M polymorphisms and alleles in extremely obese patients and controls.

\section{AGT M235T polymorphism}

The frequencies of $M M, M T$ and TT genotypes of AGT M235T polymorphism in extremely obese patients differed significantly from those in controls $(p<0.05)$. There were also statistically significant differences in the distribution of $A G T$ M235T genotypes between extremely obese patients with T2DM and controls $(p<0.05)$. The allele frequencies of all extremely obese patients and of diabetic obese patients were not statistically significantly different from those in the control group $(p>0.1)$. No significant differences were found in genotype and allele frequencies of $A G T$ M235T polymorphism between extremely obese patients without T2DM and the control group ( $p>0.1)$ (Table 2).

The crude odds ratio of morbid obesity was OR $=1.85 ; 95 \%$ CI: 1.14 - 3.02, $(p<0.05)$ for the MT genotype versus TT genotype. Similarly, the value of OR indicated that $M T$ heterozygotes had 2-fold increased risk for extreme obesity and T2DM compared to TT homozygotes, OR $=2.31 ; 95 \%$ CI: $1.29-4.15$, $(p<0.01)$, (Table 3a). The distribution of genotypes for AGT M235T did not reveal statistically significant differences between diabetic and non-diabetic obese patients $(p>0.1)$, (Table 2). In terms of $A G T$ M235T polymorphism, no significant associations with T2DM in morbid obesity were found (Table $3 \mathrm{~b}$ ).

\section{AGT T174M polymorphism}

No statistically significant differences were found in the distribution of TT, TM and MM genotypes of AGT T174M polymorphism between the control group and the study group of patients with extreme obesity ( $p>0.1$ ). The allele frequencies of the AGT gene T174M polymorphism did not differ statistically significantly in each investigated group in comparison to the control lean subjects $(p>0.1)$ (Table 2).

\section{Interactions between $A G T$ gene polymorphisms - haplotype distribution}

The M235T and T174M SNPs were in strong linkage disequilibrium $(p<0.0001)$ and formed four AGT haplotypes, termed Hap1 (235M/174T), Hap2 (235T/174T), Hap3 (235T/174M) and Hap4 $(235 \mathrm{M} / 174 \mathrm{M})$. Relative haplotype frequencies in controls and in

\begin{tabular}{|c|c|c|c|c|c|c|}
\hline & all cases & $p$ value & cases without T2DM & $p$ value & cases with T2DM & $p$ value \\
\hline $\begin{array}{l}\text { AGT M235T } \\
\text { genotypes }\end{array}$ & $\begin{array}{c}\mathrm{OR}, 95 \% \mathrm{Cl} \\
(\mathrm{n}=335)\end{array}$ & & $\begin{array}{c}\mathrm{OR}, 95 \% \mathrm{Cl} \\
(\mathrm{n}=134)\end{array}$ & & $\begin{array}{c}\mathrm{OR}, 95 \% \mathrm{Cl} \\
(\mathrm{n}=201)\end{array}$ & \\
\hline$M T$ vs $M M$ & $0.956(0.788-1.159)$ & 0.646 & $0.887(0.548-1.436)$ & 0.627 & $1.260(0.814-1.950)$ & 0.299 \\
\hline$T T$ vs $M M$ & $1.301(0.997-1.697)$ & 0.053 & $0.648(0.335-1.253)$ & 0.197 & $0.545(0.288-1.033)$ & 0.063 \\
\hline$M T$ vs $T T$ & $1.852(1.138-3.015)$ & 0.013 & $1.370(0.742-2.529)$ & 0.314 & $2.310(1.287-4.145)$ & 0.005 \\
\hline$M M$ vs TT & $1.692(0.006-1.058)$ & 0.053 & $1.544(0.798-2.986)$ & 0.197 & $1.833(0.968-3.471)$ & 0.063 \\
\hline \multicolumn{7}{|l|}{$\begin{array}{c}A G T \text { T174M } \\
\text { genotypes }\end{array}$} \\
\hline$T M$ vs TT & $1.167(0.797-1.711)$ & 0.427 & $1.088(0.670-1.767)$ & 0.734 & $1.223(0.798-1.875)$ & 0.356 \\
\hline$M M$ vs $T T$ & $1.801(0.345-9.390)$ & 0.485 & $0.876(0.078-9.790)$ & 0.915 & $2.446(0.441-13.553)$ & 0.306 \\
\hline
\end{tabular}

T2DM: Type 2 Diabetes Mellitus

Table 3a: Odds ratio (OR) of $A G T$ genotypes $(95 \% \mathrm{Cl})$ for risk of extreme obesity and T2DM.

\begin{tabular}{|c|c|c|}
\hline & T2DM in obesity & p value \\
\hline$A G T$ M235T genotypes & OR, 95\% Cl (n=335) & 0.162 \\
\hline$M T$ vs $M M$ & $1.420(0.868-2.321)$ & 0.650 \\
\hline$T T$ vs $M M$ & $0.842(0.401-1.770)$ & 0.139 \\
\hline$M T$ vs $T T$ & $1.686(0.844-3.368)$ & 0.650 \\
\hline$M M$ vs $T T$ & $1.187(0.565-2.495)$ & \\
\hline AGT $T 174 \mathrm{M}$ genotypes & & \\
\hline$T M$ vs $T T$ & $1.124(0.689-1.835)$ & 0.639 \\
\hline$M M$ vs $T T$ & $2.791(0.307-25.359)$ & 0.362 \\
\hline
\end{tabular}

T2DM: Type 2 Diabetes Mellitus

Table 3b: Odds ratio (OR) of $A G T$ genotypes $(95 \% \mathrm{Cl}$ ) for risk of T2DM in extreme obesity. 
Citation: Marta P, Tomasz F, Jan K, Przemysław A, Jacek C, et al. (2015) Angiotensinogen Gene M235T and T174M Polymorphisms in Patients with Morbid Obesity and Type 2 Diabetes Mellitus. J Diabetes Metab 6: 479. doi:10.4172/2155-6156.1000479

Page 5 of 8

extremely obese patients are shown in Table 4 . A substantial part of the genotype carrying the $235 \mathrm{M}$ allele was in that carrying $174 \mathrm{~T}$ allele (Hap1). Since the frequency of the Hap4 haplotype was $<1 \%(0.88 \%)$ and the remaining three haplotypes were sufficient to assign six haplotype pairs to about $99 \%$ of study subjects (Table 4), we excluded Hap4 from further analyses. Hap4 was found in eight Hap1Hap4 (M235M/T174M) and in two Hap4Hap3 (M235T/M174M) haplotype pairs. No haplotype pair Hap4Hap4 (M235M/M174M) was identified. The observed frequencies of six possible haplotype pairs based on these three haplotypes were consistent with the expected frequencies according to the Hardy-Weinberg equilibrium $(p>0.80)$.

The distributions of $A G T$ haplotypes in extremely obese patients did not differ significantly from those in controls $(p>0.1)$, (Table 4). Genotypic interaction within AGT M235T and T174M polymorphisms as haplotype pairs and their frequencies are presented in Table $5 \mathrm{a}$ and $5 \mathrm{~b}$. There were no significant differences in the distribution of AGT haplotype pairs between lean control subjects and extremely obese patients $(p>0.05)$ (Table 5a) and extremely obese patients without T2DM $(p>0.1)$ (Table $5 b)$.

In contrast, frequencies of haplotype pairs in diabetic extremely obese patients differed significantly from those observed in subjects without obesity $(p<0.05)$ (Table $5 b)$. Each of the six genotype combinations versus the most frequent haplotype (Hap1Hap2) as reference resulted in odds ratio (ORs) calculations. We did not find association between any of the six analysed haplotypes and morbid obesity risk (Table 5a). No haplotypes increased the risk of morbid obesity with concomitant T2DM (Table 5b). In contrast, the univariate regression analysis confirmed the negative association of the Hap2Hap3 (T235T/T174M) haplotype pair with morbid obesity OR $=0.49 ; 95 \%$ CI: $0.24-0.99,(p<0.05)($ Table 5a) and Hap2Hap2 (T235T/T174T) haplotype pair with morbid obesity and T2DM, OR $=0.34 ; 95 \% \mathrm{CI}$ : $0.14-0.85,(p<0.05)$ (Table 5b).

The genotype distribution of AGT M235T and T174M SNPs as individual haplotype pairs was comparable between extremely obese patients with T2DM and subjects with obesity and normal glucose metabolism ( $p>0.1)$. None of the AGT haplotype pairs was related to the presence of T2DM in obesity status, although univariate analysis identified the Hap2Hap2 (T235T/T174T) haplotype pair to be negatively correlated with the risk of T2DM in morbidly obese patients OR $=0.31 ; 95 \%$ CI: $0.11-0.83,(p<0.05)$ (Table $5 c$ ).

\section{Discussion}

Obesity is a complex disorder caused by the interaction of environmental factors and genetic susceptibility. The detection

\begin{tabular}{|c|c|c|c|c|c|c|c|c|c|c|}
\hline \multirow{3}{*}{ Haplotype } & \multirow{3}{*}{ M235T variant } & \multirow{3}{*}{ T174M variant } & \multirow{2}{*}{\multicolumn{2}{|c|}{$\begin{array}{c}\text { Control subjects } \\
\qquad(n=230)\end{array}$}} & \multicolumn{6}{|c|}{ Extremely obese patients } \\
\hline & & & & & \multicolumn{2}{|c|}{ all cases $(n=335)$} & \multicolumn{2}{|c|}{ cases without T2DM $(n=134)$} & \multicolumn{2}{|c|}{ cases with T2DM $(n=201)$} \\
\hline & & & $\mathrm{n}$ & $\%$ & $\mathrm{n}$ & $\%$ & $\mathrm{n}$ & $\%$ & $\mathrm{n}$ & $\%$ \\
\hline Hap 1 & M & $\mathrm{T}$ & 250 & 54.35 & 387 & 57.76 & 157 & 58.58 & 230 & 57.21 \\
\hline Hap 2 & $\mathrm{~T}$ & $\mathrm{~T}$ & 148 & 32.17 & 179 & 26.72 & 73 & 27.24 & 106 & 26.37 \\
\hline Hap 3 & $\mathrm{~T}$ & M & 60 & 13.04 & 96 & 14.33 & 36 & 13.43 & 60 & 14.93 \\
\hline Hap 4 & M & M & 2 & 0.44 & 8 & 1.19 & 2 & 0.75 & 6 & 1.49 \\
\hline$p$ value & & & \multicolumn{2}{|c|}{$0.144^{a}$} & \multicolumn{2}{|c|}{$0.521^{b}$} & \multicolumn{2}{|c|}{$0.118^{c}$} & \multicolumn{2}{|c|}{$0.815^{d}$} \\
\hline
\end{tabular}

Hap4 were excluded from analyses

$p$ values indicate differences in haplotype frequencies between groups: a controls vs. all cases; ${ }^{b}$ controls vs. cases without T2DM; ${ }^{\circ}$ Controls vs. cases with T2DM; ${ }^{d}$ Cases without T2DM vs. cases with T2DM

T: Threonine; M: Methionine; T2DM: Type 2 Diabetes Mellitus

Table 4: AGT haplotypes and their frequencies in controls and study groups.

\begin{tabular}{|c|c|c|c|c|c|c|c|c|}
\hline \multirow[t]{2}{*}{ AGT M235T } & \multirow[t]{2}{*}{ AGT T174M } & \multirow[t]{2}{*}{ Haplotype pair } & \multicolumn{2}{|c|}{ Control subjects $(n=230)$} & \multicolumn{2}{|c|}{ Extremely obese patients $(n=335)$} & \multirow[b]{2}{*}{ OR $(95 \% \mathrm{Cl})$} & \multirow[b]{2}{*}{$p$ value } \\
\hline & & & $\mathrm{n}$ & $\%$ & $\mathrm{n}$ & $\%$ & & \\
\hline MM & $\mathrm{TT}$ & Hap1 Hap1 & 65 & 28.26 & 92 & 27.46 & $0.951(0.624-1.449)$ & 0.816 \\
\hline MT & TM & Hap1 Hap3 & 35 & 15.22 & 71 & 21.19 & $1.363(0.835-2.226)$ & 0.215 \\
\hline TT & TT & Hap2 Hap2 & 21 & 9.13 & 19 & 5.67 & $0.608(0.308-1.199)$ & 0.151 \\
\hline TT & TM & Hap2 Hap3 & 22 & 9.57 & 16 & 4.78 & $0.489(0.242-0.985)$ & 0.045 \\
\hline $\mathrm{TT}$ & MM & Hap3 Hap3 & 1 & 0.43 & 4 & 1.19 & $2.688(0.295-24.471)$ & 0.380 \\
\hline
\end{tabular}

$\mathrm{p}=0.065$ (controls vs. all extremely obese cases); haplotype pairs including Hap4 were excluded from analyses

Table 5a: The frequency (\%) of individual haplotype pairs and odds ratio (OR) of AGT genotypes for risk of extreme obesity (Logistic Regression results with Hap1Hap2 as reference category)

\begin{tabular}{|c|c|c|c|c|c|c|c|c|c|c|}
\hline \multirow{3}{*}{$\begin{array}{c}A G T \\
\text { M235T }\end{array}$} & \multirow{3}{*}{$\begin{array}{c}A G T \\
\text { T174M }\end{array}$} & \multirow{3}{*}{ Haplotype pair } & \multicolumn{8}{|c|}{ Extremely obese patients } \\
\hline & & & \multicolumn{4}{|c|}{ cases without T2DM $(n=134)$} & \multicolumn{4}{|c|}{ cases with T2DM $(n=201)$} \\
\hline & & & $\mathrm{n}$ & $\%$ & OR $(95 \% \mathrm{Cl})$ & $p$ value & $\mathrm{n}$ & $\%$ & OR $(95 \% \mathrm{Cl})$ & $p$ value \\
\hline MM & $\mathrm{TT}$ & Hap1 Hap1 & 42 & 31.34 & $0.890(0.681-1.163)$ & 0.393 & 50 & 24.88 & $0.788(0.489-1.271)$ & 0.329 \\
\hline MT & TM & Hap1 Hap3 & 28 & 20.90 & $0.800(0.587-1.090)$ & 0.157 & 43 & 21.39 & $1.224(0.715-2.094)$ & 0.462 \\
\hline $\mathrm{TT}$ & $\mathrm{TT}$ & Hap2 Hap2 & 12 & 8.96 & $0.946(0.635-1.411)$ & 0.787 & 7 & 3.48 & $0.341(0.138-0.847)$ & 0.020 \\
\hline TT & TM & Hap2 Hap3 & 6 & 4.48 & $1.370(0.842-2.230)$ & 0.205 & 10 & 4.98 & $0.466(0.208-1.044)$ & 0.063 \\
\hline TT & MM & Hap3 Hap3 & 1 & 0.75 & $0.715(0.177-2.896)$ & 0.639 & 3 & 1.49 & $3.073(0.313-30.153)$ & 0.335 \\
\hline
\end{tabular}

$p=0.338$ (controls vs. cases without T2DM); $p=0.025$ (controls vs. cases with T2DM); haplotype pairs including Hap4 were excluded from analyses T2DM: Type 2 Diabetes Mellitus

Table 5b: The frequency (\%) of individual haplotype pairs and odds ratio (OR) of AGT genotypes for risk of extreme obesity without and with T2DM . (Logistic Regression results with Hap1Hap2 as reference category). 


\begin{tabular}{|c|c|c|c|c|c|}
\hline \multirow{2}{*}{$A G T$ M235T } & AGT T174M & Haplotype pair & \multicolumn{3}{|c|}{ Extremely obese patients with T2DM (n = 201) } \\
\cline { 3 - 6 } & & Hap1 Hap1 & 50 & 24.88 & $0.624(0.360-1.084)$ \\
\hline MM & TT & Hap1 Hap3 & 43 & 21.39 & $0.805(0.441-1.471)$ \\
\hline MT & TM & Hap2 Hap2 & 7 & 3.48 & $0.306(0.112-0.834)$ \\
\hline TT & TT & Hap2 Hap3 & 10 & 4.98 & 0.481 \\
\hline TT & TM & Hap3 Hap3 & 3 & 1.49 & $1.573(0.159-15.583)$ \\
\hline TT & MM & & 0.021 & 0.806 \\
\hline
\end{tabular}

$p=0.191$ (cases without T2DM vs. cases with T2DM)

T2DM: Type 2 Diabetes Mellitus

Table 5c: The frequency (\%) of individual haplotype pairs and odds ratio (OR) of $A G T$ genotypes for risk of diabetes mellitus in extreme obesity. (Logistic Regression results with Hap1Hap2 as reference category)

of functional mutations in genes involved in the process of fat accumulation may help identify specific targets for pharmacologic intervention $[4,5]$. There is considerable amount of data from animal and human experiments suggesting that the RAS is involved in the regulation of body fat and it is therefore a subject of interest in the studies of obesity [6].

The present research study was performed to analyze the relationship of the angiotensinogen gene M235T and T174M polymorphisms with morbid obesity and to investigate the effect of $A G T$ gene variations on the development of morbid obesity and type 2 diabetes mellitus in a sample of Polish population. We observed a statistically significant difference in the distribution of AGT M235T genotypes between subjects without obesity (control group) and patients with extreme obesity $(p<0.05)$. This may primarily be the result of higher frequency of $M T$ genotype and lower occurrence of $T T$ genotype observed in all extremely obese patients compared to the control group. MT heterozygotes had almost twice higher risk of extreme obesity in relation to TT homozygotes $(p<0.05)$. The relative risk for extreme obesity and coexisting T2DM associated with $M T$ genotype was nearly 2,5 -fold higher in relation to TT genotype $(p<0.01)$. In contrast, we could not show any significant association between $A G T \mathrm{M} 235 \mathrm{~T}$ polymorphism and the risk of T2DM in morbidly obese patients ( $p>0.1)$. Nevertheless, although we assumed that our observation of an association between $M T$ heterozigosity and morbid obesity was a real observation rather than a chance finding, it should be emphasized that we were not able to demonstrate any association between AGT M235T genotypes and body mass index, fasting glucose or prevalence of T2DM in either study group.

It is well-known that obesity is the leading risk factor for type 2 diabetes and that many diabetic patients are obese. Activation of the RAS in obesity strongly correlates to metabolic disease and it is hypothesized that it is the principle cause of diabetes mellitus [36]. In this regard, the results of our study clearly indicate that genetic predisposition to type 2 diabetes may be modulated according to obesity status. The gathered data underline complex correlations between increased body fat and insulin resistance [36]. These data also allow to suppose that insulin resistance and associated with it compensatory hyperinsulinemia together with activated RAS create a mechanism of several positive feedback pathways which not only result from obesity but also contribute to it $[6,10,11,36]$. With respect to the $A G T$ gene variation in obesity, the present study was in line with the results of other investigators [37], who suggested that polymorphisms of AGT gene might contribute to the risk of T2DM independently and/ or in an interactive manner according to the presence or absence of obesity. Our results have shown that the AGT M235T genotype alone could account for the increased probability of developing morbid obesity and obesity with T2DM. Mehri et al. [24] reported that AGT M235T genotype was associated with an increased susceptibility to $\mathrm{T} 2 \mathrm{DM}$, only in the presence of obesity. The risk of developing diabetes seemed to be higher for TT genotype and T allele compared to $M M$ genotype and $M$ allele [24]. In another study, the TT genotype of AGT M235T gene polymorphism was linked to visceral obesity and was associated with the risk for obesity-related diabetes mellitus [25]. Results of some studies support the notion that the AGT M235T and T174M polymorphisms could be a risk factor for central obesity in hypertensive patients [26,27]. In the study carried out in a white population, a significant association was found between $A G T$ gene polymorphisms and insulin sensitivity and the genotype effect was the strongest in hypertensive and obese individuals. Moreover, this finding was strengthened by haplotype analysis with AGT M235T implication [28]. Some studies failed to find the relationship between AGT M235T and obesity $[21,29,30]$.

In the present study, we found no evidence of association between the polymorphic variants of the AGT T174M polymorphism and the risk for extreme obesity and associated T2DM $(p>0.1)$. However, among all extremely obese patients and in obese patients with T2DM, a higher proportion of individuals was found to have TM and $M M$ genotypes as compared to lean subjects. In a sample of homogenous population of Majorca the T174T genotype showed a significant negative association with obesity, OR $=0.41 ; 95 \%$ CI: $0.18-0.90,(p=0.03)$ [23]. We believe that the analysis of haplotypes may provide a more precise estimation of the effects of genetic variants on a phenotype than single markers [38]. Therefore, we evaluated associations between haplotypes formed by the two investigated in our study AGT polymorphisms and morbid obesity and T2DM.

Although, the AGT T174M polymorphism was not associated with morbid obesity and concomitant diabetes mellitus, we observed in haplotype pairs distribution analysis that it could exert an influence on susceptibility to excessive body weight and impaired glucose metabolism. Our results show that none of the six investigated haplotype pairs predisposed to the risk of obesity and obesity with its metabolic complications. On the other hand, we observed that the Hap2Hap3 (T235T/T174M) haplotype pair could protect against morbid obesity and carriers of Hap2Hap2 (T235T/T174T) haplotype pair had decreased risk for morbid obesity and concomitant T2DM. It is difficult to compare our results with the findings of other investigators on the genetic susceptibility to morbid obesity, since there have been published no reports related to the role of the AGT gene polymorphisms and their interaction in the development of extreme obesity. In our study, the investigated group included only patients with extreme obesity (mean BMI $\sim 45 \mathrm{~kg} / \mathrm{m}^{2}$ ). We assumed that in extreme obesity, the contribution of genetic factors to the pathogenesis of excessive fat accumulation could be substantially higher than in the lower class of obesity. Genetics and physiology studies provided insight into a possible mechanism underlying our finding of an association of the AGT gene polymorphisms with obesity and diabetes mellitus $[9,13]$. Molecular studies demonstrated a relationship between variants 
of the $A G T$ gene, specifically the M235T polymorphism and AGT gene expression, that was associated with plasma AGT levels and essential hypertension [16,17,39]. Renner et al. [39] observed that a single haplotype, consisting of $235 \mathrm{~T}$ and $174 \mathrm{~T}$ allele, accounted for approx. $8 \%$ increase in plasma angiotensinogen concentration and this association was stronger than that of $A G T$ gene single polymorphism. In that case, it can be assumed that in our investigations subjects with Hap2Hap2 haplotype pair (T235T/T174T) could have the highest plasma AGT level, and just these subjects had, in our study, a decreased risk for obesity and diabetes mellitus. Hap2Hap2 haplotype pair had protective effect on developing T2DM in extremely obese patients. On the basis of the above mentioned observations $[9,13]$, one can think that subjects carrying at least one Hap2 haplotype and genetically determined higher AGT levels would have higher risk for the development of obesity and T2DM. Such correlation has not been confirmed in our study.

AGT synthesis in the subcutaneous adipose tissue was regulated by insulin, so it is likely that AGT might affect insulin resistance [40]. Basing on this evidence, we could suspect that our morbidly obese subjects had elevated level of AGT. The results of experimental studies concerning the role of RAS in the metabolism of adipocytes could partly explain the observed in our study possible effect of $A G T$ gene polymorphisms on the development of extreme obesity and T2DM. We speculate that this phenomenon could be linked to direct modification of the adipose tissue by Ang II and may be associated with changes in fat cell size [6]. Some potential limitations should be considered in our study. Our study has a relatively small sample size in comparison with the genome-wide association approach. However, our research was conducted with a well phenotyped group of patients and representative of our population. Further studies with larger samples and different populations are necessary in order to confirm our findings. Another limitation to this study is that we used two missense AGT polymorphisms located in the AGT coding region for haplotype building, but we did not include polymorphisms of G-6A and $\mathrm{A}-20 \mathrm{C}$ in promoter region that are in linkage disequilibrium with the $235 \mathrm{~T}$ and $174 \mathrm{M}$ alleles, respectively [15,41-44]. We did not analyse the relationship between the $A G T$ gene variants and angiotensinogen levels which may also be regarded as a limitation of the study. An interaction between lifestyle, physical activity and familial history of extreme obesity could be a confounding factor and these were not investigated. The findings of the present study have to be interpreted within the context of its limitations.

Further studies are needed to clarify the relation between AGT gene polymorphisms and obesity and to explore the putative mechanism(s) in the case of an increased susceptibility of AGT M235T MT carriers to morbid obesity and T2DM.

\section{Conclusions}

To conclude, the results of our study suggest that M235T, but not the T174M polymorphism of the angiotensinogen $(A G T)$ gene, may be linked with extreme obesity and T2DM in the investigated group of patients. Our study has shown for the first time that the risk for extreme obesity, irrespective of T2DM, was lower in Hap2Hap3 haplotype pair carriers. The Hap2Hap2 haplotype pair was protective against morbid obesity with T2DM, and also against T2DM development in extremely obese patients.

\section{Acknowledgments}

We thank Mrs Beata Błaszkiewicz and Mrs Jadwiga Kacprzak for their excellent technical and laboratory assistance.

\section{References}

1. Bray GA, Bellanger T (2006) Epidemiology, trends, and morbidities of obesity and the metabolic syndrome. Endocrine 29: 109-117.

2. Bays HE, González-Campoy JM, Henry RR, Bergman DA, Kitabchi AE, et al (2008) Is adiposopathy (sick fat) an endocrine disease? Int J Clin Pract 62 1474-1483.

3. Koebnick C, Smith N, Huang K, Martinez MP, Clancy HA, et al. (2012) The prevalence of obesity and obesity-related health conditions in a large multiethnic cohort of young adults in California. Ann Epidemiol 22: 609-616.

4. Hetherington MM, Cecil JE (2010) Gene-environment interactions in obesity. Forum Nutr 63: 195-203

5. Dahlman I, Arner P (2007) Obesity and polymorphisms in genes regulating human adipose tissue. Int J Obes (Lond) 31: 1629-1641.

6. Mathai ML, Chen N, Cornall L, Weisinger RS (2011) The role of angiotensin in obesity and metabolic disease. Endocr Metab Immune Disord Drug Targets 11: 198-205.

7. Karlsson C, Lindell K, Ottosson M, Sjöström L, Carlsson B, et al. (1998) Human adipose tissue expresses angiotensinogen and enzymes required for its conversion to angiotensin II. J Clin Endocrinol Metab 83: 3925-3929.

8. Engeli S, Gorzelniak K, Kreutz R, Runkel N, Distler A, et al. (1999) Coexpression of renin-angiotensin system genes in human adipose tissue. $J$ Hypertens 17: 555-560.

9. Goossens GH, Blaak EE, van Baak MA (2003) Possible involvement of the adipose tissue renin-angiotensin system in the pathophysiology of obesity and obesity-related disorders. Obes Rev 4: 43-55.

10. Kalupahana NS, Moustaid-Moussa N (2012) The renin-angiotensin system: a link between obesity, inflammation and insulin resistance. Obes Rev 13: 136149 .

11. Yvan-Charvet L, Quignard-Boulangé A (2011) Role of adipose tissue reninangiotensin system in metabolic and inflammatory diseases associated with obesity. Kidney Int 79: 162-168.

12. Jeunemaitre X, Inoue I, Williams C, Charru A, Tichet J, et al. (1997) Haplotypes of angiotensinogen in essential hypertension. Am J Hum Genet 60: 1448-1460.

13. Bloem LJ, Foroud TM, Ambrosius WT, Hanna MP, Tewksbury DA, et al. (1997) Association of the angiotensinogen gene to serum angiotensinogen in blacks and whites. Hypertension 29: 1078-1082.

14. Jeunemaitre $X$, Soubrier $F$, Kotelevtsev YV, Lifton RP, Williams CS, et al. (1992) Molecular basis of human hypertension: role of angiotensinogen. Cell 71: 169-180.

15. Mohana VU, Swapna N, Surender RS, Vishnupriya S, Padma T (2012) Genderrelated association of AGT gene variants (M235T and T174M) with essential hypertension--a case-control study. Clin Exp Hypertens 34: 38-44.

16. Miller JA, Scholey JW (2004) The impact of renin-angiotensin system polymorphisms on physiological and pathophysiological processes in humans. Curr Opin Nephrol Hypertens 13: 101-106.

17. Sethi AA, Nordestgaard BG, Tybjaerg-Hansen A (2003) Angiotensinogen gene polymorphism, plasma angiotensinogen, and risk of hypertension and ischemic heart disease: a meta-analysis. Arterioscler Thromb Vasc Biol 23: 1269-1275.

18. Strazzullo P, lacone R, lacoviello L, Russo O, Barba G, et al. (2003) Genetic variation in the renin-angiotensin system and abdominal adiposity in men: the Olivetti Prospective Heart Study. Ann Intern Med 138: 17-23.

19. Yang JK, Zhou JB, Xin Z, Zhao L, Yu M, et al. (2010) Interactions among related genes of renin-angiotensin system associated with type 2 diabetes. Diabetes Care 33: 2271-2273.

20. Marco J, Zabay JM, Garcia-Marco MA, Gomez G, Mulet JM, et al. (2005) Angiotensinogen gene T174M polymorphism: opposite relationships with essential hypertension and obesity in a homogeneous population from Majorca (Baleric Islands, Spain) Nefrologia 25: 629-636.

21. Mehri S, Koubaa N, Hammami S, Mahjoub S, Chaaba R, et al. (2010) Genotypic interactions of renin-angiotensin system genes with diabetes type 2 in a Tunisian population. Life Sci 87: 49-54.

22. Takakura Y, Yoshida T, Yoshioka K, Umekawa T, Kogure A, et al. (2006) Angiotensinogen gene polymorphism (Met235Thr) influences visceral obesity and insulin resistance in obese Japanese women. Metabolism 55: 819-824. 
Citation: Marta P, Tomasz F, Jan K, Przemysław A, Jacek C, et al. (2015) Angiotensinogen Gene M235T and T174M Polymorphisms in Patients with Morbid Obesity and Type 2 Diabetes Mellitus. J Diabetes Metab 6: 479. doi:10.4172/2155-6156.1000479

Page 8 of 8

23. Procopciuc LM, Sitar-Taut A, Pop D, Sitar-Taut DA, Olteanu I, et al. (2010) Renin angiotensin system polymorphisms in patients with metabolic syndrome (MetS). Eur J Intern Med 21: 414-418.

24. Vasku A, Soucek M, Tschöplová S, Stejskalová A (2002) An association of BMI with A (-6) G, M235T and T174M polymorphisms in angiotensinogen gene in essential hypertension. J Hum Hypertens 16: 427-430.

25. Underwood PC, Sun B, Williams JS, Pojoga LH, Raby B, et al. (2011) The association of the angiotensinogen gene with insulin sensitivity in humans: a tagging single nucleotide polymorphism and haplotype approach. Metabolism 60: $1150-1157$

26. Cooper R, McFarlane-Anderson N, Bennett FI, Wilks R, Puras A, et al. (1997) $\mathrm{ACE}$, angiotensinogen and obesity: a potential pathway leading to hypertension. J Hum Hypertens 11: 107-111.

27. Niemiec P, Zak I, Wita K (2008) The M235T polymorphism of the AGT gene modifies the risk of coronary artery disease associated with the presence of hypercholesterolemia. Eur J Epidemiol 23: 349-354.

28. Pacholczyk M, Ferenc T, Kowalski J, Adamczyk P, Chojnowski J, et al. (2013) Association of angiotensin-converting enzyme and angiotensin II type I receptor gene polymorphisms with extreme obesity in Polish individuals. DNA Cell Biol 32: $435-442$.

29. American Diabetes Association (2014) Diagnosis and classification of diabetes mellitus. Diabetes Care 37: S64-S71.

30. (2000) Obesity: preventing and managing the global epidemic. Report of a WHO consultation. World Health Organ Tech Rep Ser 894: i-xii, 1-253.

31. Lizanecz E, Pásztor ET, Mohácsi A, Papp Z, Edes I, et al. (2006) Mistyping of angiotensinogen M235T alleles. Hypertens Res 29: 197-201.

32. Gutiérrez C, Vendrell J, Pastor R, Llor C, Aguilar C, et al. (1997) Angiotensin I-converting enzyme and angiotensinogen gene polymorphisms in non-insulindependent diabetes mellitus. Lack of relationship with diabetic nephropathy and retinopathy in a Caucasian Mediterranean population. Metabolism 46: 976-980.

33. Kahn BB, Flier JS (2000) Obesity and insulin resistance. J Clin Invest 106 473-481.
34. Lin E, Pei D, Huang YJ, Hsieh CH, Wu LS (2009) Gene-gene interactions among genetic variants from obesity candidate genes for nonobese and obese populations in type 2 diabetes. Genet Test Mol Biomarkers 13: 485-493.

35. Watkins WS, Hunt SC, Williams GH, Tolpinrud W, Jeunemaitre X, et al (2010) Genotype-phenotype analysis of angiotensinogen polymorphisms and essential hypertension: the importance of haplotypes. J Hypertens 28: 65-75.

36. Zhao H, Pfeiffer R, Gail MH (2003) Haplotype analysis in population genetics and association studies. Pharmacogenomics 4: 171-178.

37. Luther JM, Brown NJ (2011) The renin-angiotensin-aldosterone system and glucose homeostasis. Trends Pharmacol Sci 32: 734-739.

38. Renner W, Nauck M, Winkelmann BR, Hoffmann MM, Scharnagl $\mathrm{H}$, et al. (2005) Association of angiotensinogen haplotypes with angiotensinogen levels but not with blood pressure or coronary artery disease: the Ludwigshafen Risk and Cardiovascular Health Study. J Mol Med (Berl) 83: 235-239.

39. Giacchetti G, Faloia E, Sardu C, Camilloni MA, Mariniello B, et al. (2000) Gene expression of angiotensinogen in adipose tissue of obese patients. Int $\mathrm{J}$ Obes Relat Metab Disord 24 Suppl 2: S142-143.

40. Harte A, McTernan P, Chetty R, Coppack S, Katz J, et al. (2005) Insulinmediated upregulation of the renin angiotensin system in human subcutaneous adipocytes is reduced by rosiglitazone. Circulation 111: 1954-1961.

41. Mogi M, Iwai M, Horiuchi M (2006) Emerging concept of adipogenesis regulation by the renin-angiotensin system. Hypertension 48: 1020-1022.

42. Janke J, Engeli S, Gorzelniak K, Luft FC, Sharma AM (2002) Mature adipocytes inhibit in vitro differentiation of human preadipocytes via angiotensin type 1 receptors. Diabetes 51: 1699-1707.

43. Schling P, Löffler G (2001) Effects of angiotensin II on adipose conversion and expression of genes of the renin-angiotensin system in human preadipocytes. Horm Metab Res 33: 189-195.

44. Morgan L, Broughton Pipkin F, Kalsheker N (1996) DNA polymorphisms and linkage disequilibrium in the angiotensinogen gene. Hum Genet 98: 194-198. 